\title{
An Elevated Platelet-to-Lymphocyte Ratio Predicts Poor Prognosis and Clinicopathological Characteristics in Patients with Colorectal Cancer: A Meta-Analysis
}

\author{
Xuan-zhang Huang, Wen-jun Chen, Xi Zhang, Cong-cong Wu, Chao-ying Zhang, \\ Shuang-shuang Sun, and Jian Wu \\ Department of Chemotherapy and Radiotherapy, The Second Affiliated Hospital and Yuying Children's Hospital of Wenzhou \\ Medical University, 109 Xueyuan West Road, Lucheng District, Wenzhou 325027, China \\ Correspondence should be addressed to Jian Wu; wzzjian1@163.com
}

Received 2 November 2016; Accepted 2 April 2017; Published 26 April 2017

Academic Editor: Paola Gazzaniga

Copyright (c) 2017 Xuan-zhang Huang et al. This is an open access article distributed under the Creative Commons Attribution License, which permits unrestricted use, distribution, and reproduction in any medium, provided the original work is properly cited.

Background. The aims of this study were to evaluate the clinicopathological and prognostic values of platelet-to-lymphocyte ratio (PLR) in colorectal cancer (CRC). Methods. The PubMed and Embase databases and the references of relevant studies were systematically searched. This study was performed with hazard ratios (HRs) and odd ratios (ORs) with corresponding 95\% confidence intervals (CIs) as effect measures. Results. Our results indicated that elevated PLR was associated with poor overall survival $(\mathrm{HR}=1.46,95 \% \mathrm{CI}=1.23-1.73)$, disease-free survival $(\mathrm{HR}=1.64,95 \% \mathrm{CI}=1.17-2.30)$, cancer-specific survival $(\mathrm{HR}=1.30,95 \% \mathrm{CI}=1.12-1.51)$, and recurrence-free survival $(\mathrm{HR}=1.38,95 \% \mathrm{CI}=1.09-1.74)$ in CRC. For the clinicopathological characteristics, our results indicated that there were differences in the rate of elevated PLR between stages III/IV and I/II groups $(\mathrm{OR}=1.38,95 \% \mathrm{CI}=1.01-1.88)$, pT3/T4 and pT1/T2 groups $(\mathrm{OR}=1.82$, 95\% $\mathrm{CI}=1.03-3.20)$, and poor differentiation and moderate/well differentiation $(\mathrm{OR}=2.59,95 \% \mathrm{CI}=1.38-4.84)$. Conclusions . Our results indicated that elevated PLR predicted poor prognosis and clinicopathological characteristics in CRC and PLR is a convenient and low-cost blood-derived prognostic marker for CRC.

\section{Introduction}

Colorectal cancer (CRC) is the third most frequently diagnosed cancer in males and the second in females [1]. Tumor metastasis and recurrence still remain the major cause of mortality. However, there is a lack of precise biomarkers for predicting prognosis in CRC that can be used for individualized treatment. Thus, it is clinically important to find reliable prognostic markers for cancer treatment.

Bodies of evidence have shown that the interactions between tumor and host-derived microenvironments, such as inflammation, immune response, and coagulation status, play an important role in tumor progression and prognosis [2-4]. Severe inflammatory responses could result in an imbalance of the immune response, promoting tumor progression [3-5]. Recently, as a convenient and costeffective blood-derived marker, the platelet-to-lymphocyte ratio (PLR), which takes into account the inflammatory response, immune response, and coagulation status, has been widely investigated as a useful prognostic factor in various solid cancers [6, 7]. However, the prognostic values of PLR in CRC are controversial and have not been confirmed [8-12]. Furthermore, whether the PLR could predict the clinicopathological characteristics of CRC is also unclear.

The purposes of the present study were to use a metaanalysis to quantitatively and comprehensively summarize the clinicopathological and prognostic significance of the PLR in CRC.

\section{Materials and Methods}

2.1. Literature Search. PubMed and Embase databases were systematically searched for all relevant studies (up to February 2016). Moreover, the reference lists of all relevant studies and 
reviews were also manually searched to identify any potentially eligible studies. The following search terms were used: "platelet-to-lymphocyte ratio", "platelet-lymphocyte ratio", "platelet to lymphocyte ratio", "colorectal cancer", "colon cancer", and "rectal cancer".

2.2. Eligibility Criteria. Studies were included in our metaanalysis if they met all of the following inclusion criteria: (1) the included patients were diagnosed as CRC, (2) the outcome of interest was the clinicopathological and/or prognostic relationship between PLR and CRC, and (3) the outcome measures of interest could be extracted directly or could be calculated from the published data indirectly. If several duplicated studies based on the same population met the inclusion criteria, only the most informative study was included in our meta-analysis.

2.3. Data Extraction and Quality Assessment. Eligible studies were reviewed, and data of interest were extracted by two reviewers, independently. The following data were extracted: first author, publication year, country, population characteristics, tumor clinicopathological characteristics, sampling time, cut-off value, rate of elevated PLR, and prognostic value of PLR (overall survival (OS), disease-free survival (DFS), cancerspecific survival (CSS), and recurrence-free survival (RFS)).

The quality of the included studies was evaluated using the Newcastle-Ottawa Scale (NOS) criteria [13]. In addition, any disagreements on data extraction and/or quality assessment were resolved through comprehensive discussion.

2.4. Statistical Analysis. Hazard ratios (HRs) and odds ratios (ORs) with corresponding 95\% confidence intervals (CIs) were used as measures to summarize the relationship between PLR and prognosis and between PLR and tumor clinicopathological characteristics, respectively. HRs and 95\% CIs were extracted directly, or they were calculated from available data using the methods designed by Tierney et al. [14]. Subgroup analyses were conducted stratified by sampling time, metastatic status, sample size, cut-off value, country, and study quality. We also conducted subgroup analysis based on study analysis type in the primary studies to explore the impact of multivariable and univariable analysis.

The heterogeneity among the studies was assessed using the $I^{2}$ statistics and Cochran $Q$ test. A random effects model was used to pool measures if substantial heterogeneity existed; otherwise, a fixed effects model was used. A metaregression analysis was conducted to explore potential variables that contributed heterogeneity or dominated results [15]. Begg's and Egger's tests were used to evaluate publication bias, and a trim-and-fill analysis was performed to assess the effect of publication bias if a significant publication bias existed [16].

All statistical analyses were performed using Stata software version 12.0 (Stata Corporation, College Station, TX, USA). A two-sided $P$ value $<0.05$ was considered statistically significant.

\section{Results}

3.1. Study Selection and Study Characteristics. A total of 191 studies were initially identified from the literature search,

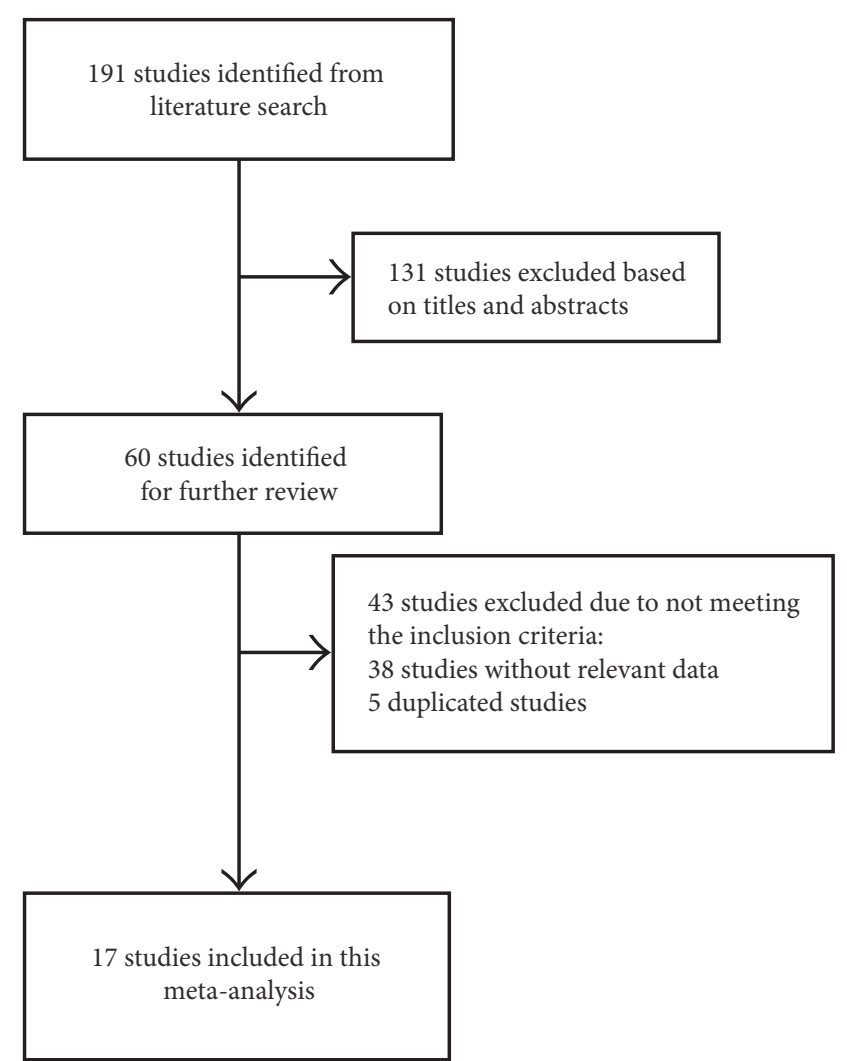

Figure 1: Flow diagram showing the selection process for the included studies.

and 131 studies were excluded after reviewing the titles and abstracts. After a full-text review, 38 studies were excluded. Finally, 17 studies were included in our meta-analysis (Figure 1) [8-12, 17-28].

The 17 eligible studies included 4968 CRC patients (mean sample size: 292; median and range of sample size: 243 and 110-624). The studies were from the USA, the United Kingdom, Austria, Canada, China, Korea, Hungary, and Japan, and the year of publication ranged from 2011 to 2015. The baseline characteristics and quality of studies are summarized in Table 1.

\section{Impact of PLR on Survival}

4.1. PLR and OS. The pooled estimated HRs indicate that elevated PLR was associated with poor OS in CRC $(\mathrm{HR}=1.46$, $95 \% \mathrm{CI}=1.23-1.73$, Figure 2 ). Including studies only assessing preoperative PLR, our results also indicate that elevated PLR predicted a poor OS $(\mathrm{HR}=1.61,95 \% \mathrm{CI}=1.28-2.02)$.

We conducted subgroup analyses stratified by cut-off value and sample size, and the results confirmed PLR as a prognostic factor for OS: cut-off value (cut-off $>150$ : $\mathrm{HR}=1.60,95 \% \mathrm{CI}=1.18-2.17$; cut-off $\leq 150$ : $\mathrm{HR}=1.33$, $95 \% \mathrm{CI}=1.08-1.64$ ) and sample size (sample size $\geq 250$ : $\mathrm{HR}=1.36,95 \% \mathrm{CI}=1.01-1.82$; sample size $<250: \mathrm{HR}=1.53$, $95 \% \mathrm{CI}=1.32-1.77)$. As shown by the subgroup analyses stratified by distant metastasis status, study quality, study analysis type, and country, the prognostic effect of PLR on OS was also confirmed (Table 2). 


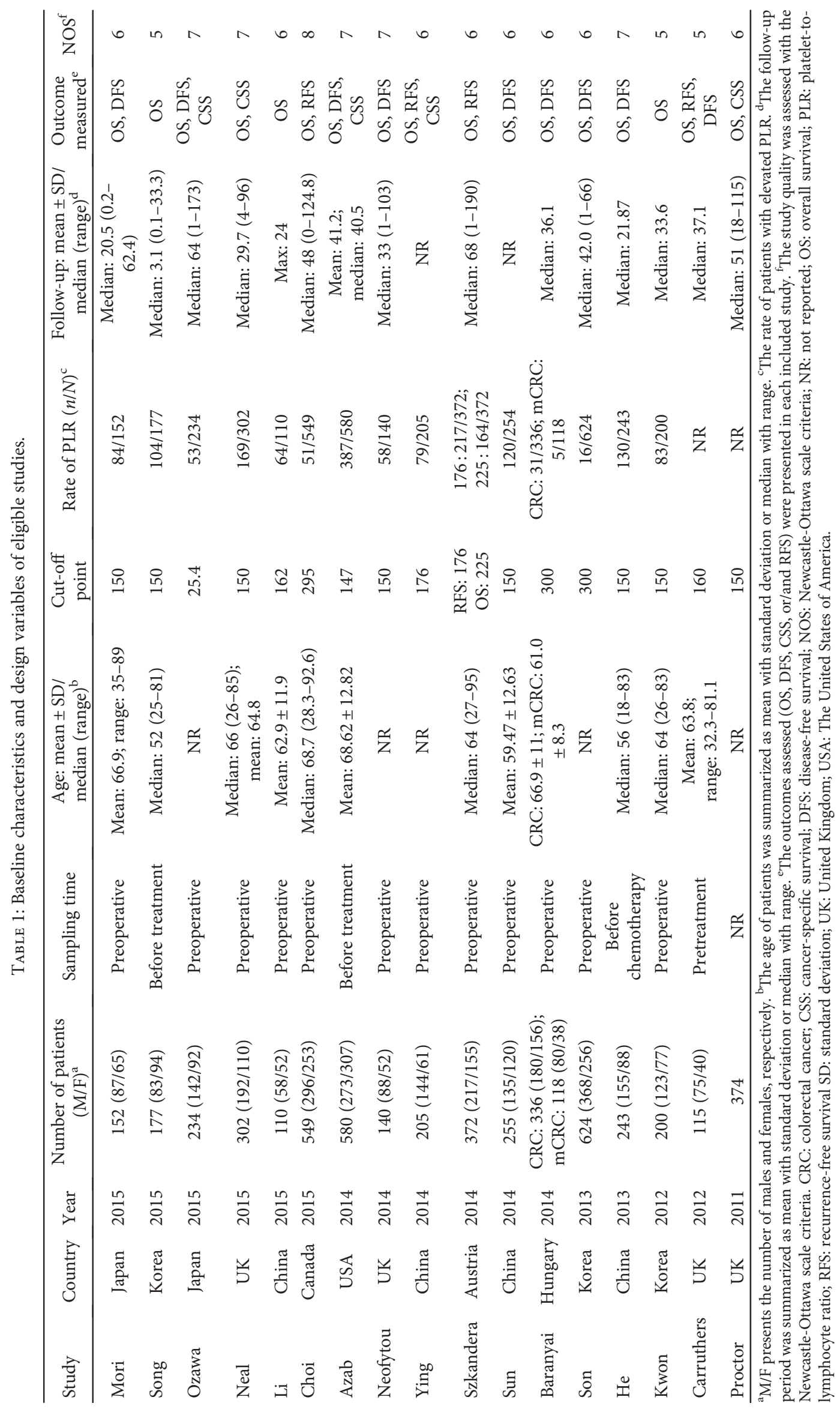




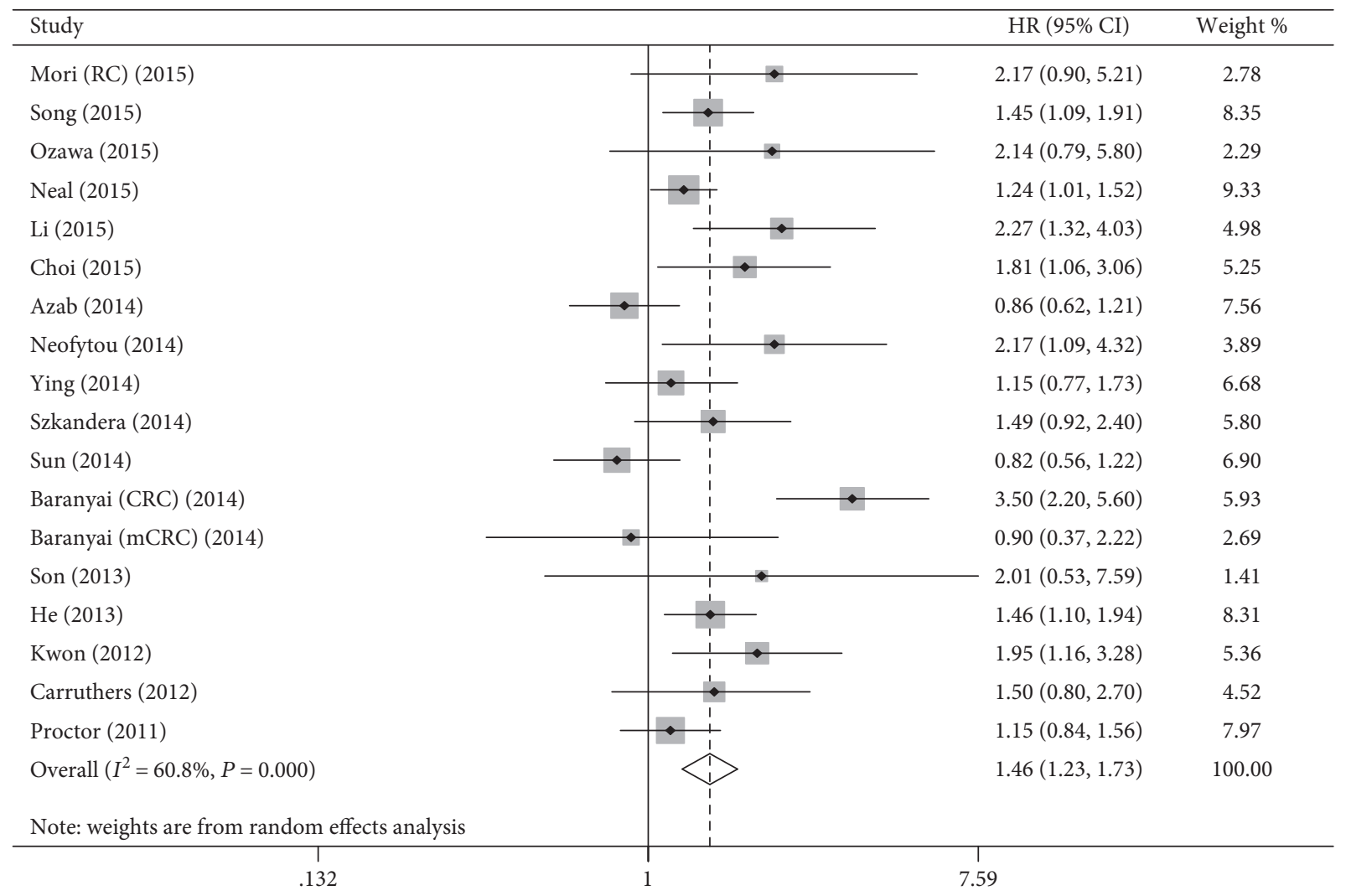

FIGURE 2: The estimated hazard ratio (HR) was summarized for the relationship between platelet-to-lymphocyte ratio and overall survival.

4.2. PLR and DFS. The poor prognosis for DFS in CRC was indicated by the elevated PLR $(\mathrm{HR}=1.64,95 \% \mathrm{CI}=1.17$ 2.30 , Figure 3(a)). Moreover, the result of subgroup analysis for preoperative PLR was similar, predicting poor DFS $(\mathrm{HR}=1.78,95 \% \mathrm{CI}=1.12-2.83)$.

The subgroup analysis based on cut-off value $\leq 150$ $(\mathrm{HR}=1.49,95 \% \mathrm{CI}=1.03-2.14)$ and metastasis positive $(\mathrm{HR}=1.76,95 \% \mathrm{CI}=1.23-2.51)$ provided a similar result. In addition, the results of subgroup analyses based on study analysis type, country, and study quality confirmed that elevated PLR tended towards worse DFS (Table 2).

4.3. PLR and CSS. Our results indicate that CCS was worse in CRC with elevated PLR compared with those with low PLR $(\mathrm{HR}=1.30,95 \% \mathrm{CI}=1.12-1.50$, Figure 3(b)). Similarly, the results of preoperative PLR showed that elevated PLR was associated with worse CSS ( $\mathrm{HR}=1.26,95 \%$ $\mathrm{CI}=1.04-1.52)$. We observed a similar result in the subgroup analyses stratified by multivariable analysis type, univariable analysis type, sample size $\geq 250$, cut-off value $\leq 150$, and NOS $\geq 6$ (Table 2 ).

4.4. PLR and RFS. All the studies used a cut-off value $>150$ and preoperative PLR. RFS was worse in patients with elevated PLR compared with those with low PLR $(\mathrm{HR}=1.38$, $95 \% \mathrm{CI}=1.09-1.74$, Figure 3(c)). As was shown by subgroup analyses on metastasis status, sample size, country, and study quality, similar results were observed (Table 2).

\section{Correlation of PLR with Clinicopathological Characteristics}

The meta-analysis of relevant studies on TNM stage indicated a higher rate of elevated PLR in the stage III/IV group relative to the stage I/II group $(\mathrm{OR}=1.38,95 \% \mathrm{CI}=1.01-1.88)$, as well as stage II-IV group relative to the stage I group $(\mathrm{OR}=2.77$, $95 \% \mathrm{CI}=1.87-4.12)$. The rate of elevated PLR was different between the $\mathrm{pT} 3 / \mathrm{T} 4$ and $\mathrm{pT} 1 / \mathrm{T} 2$ groups $(\mathrm{OR}=1.82,95 \%$ $\mathrm{CI}=1.03-3.20)$ and poor differentiation and moderate/well differentiation $(\mathrm{OR}=2.59,95 \% \mathrm{CI}=1.38-4.84)$. However, we could not observe an association between lymph node metastasis and PLR $(\mathrm{OR}=1.16,95 \% \mathrm{CI}=0.86-1.57)$, lymphatic invasion and PLR $(\mathrm{OR}=1.48,95 \% \mathrm{CI}=0.88-2.46)$, and venous invasion and $\mathrm{PLR}(\mathrm{OR}=1.31,95 \% \mathrm{CI}=0.79-2.17)$.

\section{Publication Bias and Metaregression}

Begg's and Egger's tests showed no substantial publication bias, except in the HRs for DFS. And the funnel plots for analyses are shown in Figure 4. The trim-and-fill analyses indicated that there might be three unpublished or missing studies existing in the meta-analysis of DFS; however, the association between PLR and DFS was still statistically significant even if the three studies were published, indicating that publication bias could not impact on the results for DFS. Our metaregression analysis suggested that sampling time, metastatic status, sample size, cut-off value, country, and study 
TABLE 2: Results of subgroup analyses for prognostic significance of platelet-to-lymphocyte ratio.

\begin{tabular}{|c|c|c|c|c|}
\hline & Overall survival & Disease-free survival & Cancer-specific survival & Recurrence-free survival \\
\hline Overall & $\begin{array}{c}\mathrm{HR}=1.46(1.23-1.73) \\
I^{2}=60.8 \%\end{array}$ & $\begin{array}{c}\mathrm{HR}=1.64(1.17-2.30) \\
I^{2}=73.5 \%\end{array}$ & $\begin{array}{c}\mathrm{HR}=1.30(1.12-1.50) \\
I^{2}=0.0 \%\end{array}$ & $\begin{array}{c}\mathrm{HR}=1.38(1.09-1.74) \\
I^{2}=20.7 \%\end{array}$ \\
\hline \multicolumn{5}{|l|}{ Sampling time } \\
\hline Preoperative & $\begin{array}{c}\mathrm{HR}=1.61(1.28-2.02) \\
I^{2}=61.4 \%\end{array}$ & $\begin{array}{c}\mathrm{HR}=1.78(1.12-2.83) \\
I^{2}=77.3 \%\end{array}$ & $\begin{array}{c}\mathrm{HR}=1.26(1.04-1.52) \\
I^{2}=33.3 \%\end{array}$ & $\begin{array}{c}\mathrm{HR}=1.38(1.09-1.74) \\
I^{2}=20.7 \%\end{array}$ \\
\hline \multicolumn{5}{|l|}{ Metastatic status } \\
\hline M1 & $\begin{array}{c}\mathrm{HR}=1.40(1.23-1.60) \\
I^{2}=26.6 \%\end{array}$ & $\begin{array}{c}\mathrm{HR}=1.76(1.23-2.51) \\
I^{2}=0.0 \%\end{array}$ & I & / \\
\hline M0 & $\begin{array}{c}\mathrm{HR}=1.63(1.15-2.30) \\
I^{2}=68.4 \%\end{array}$ & $\begin{array}{c}\mathrm{HR}=1.82(1.03-3.21) \\
I^{2}=80.7 \%\end{array}$ & $\begin{array}{c}\mathrm{HR}=1.75(0.59-5.17) \\
I^{2}=66.2 \%\end{array}$ & $\begin{array}{c}\mathrm{HR}=1.38(1.09-1.74) \\
I^{2}=20.7 \%\end{array}$ \\
\hline \multicolumn{5}{|l|}{ Sample size } \\
\hline$\geq 250$ & $\begin{array}{c}\mathrm{HR}=1.36(1.01-1.83) \\
I^{2}=77.3 \%\end{array}$ & $\begin{array}{c}\mathrm{HR}=1.46(0.80-2.64) \\
I^{2}=85.3 \%\end{array}$ & $\begin{array}{c}\mathrm{HR}=1.30(1.11-1.52) \\
I^{2}=0.0 \%\end{array}$ & $\begin{array}{c}\mathrm{HR}=1.67(1.21-2.29) \\
I^{2}=0.0 \%\end{array}$ \\
\hline$<250$ & $\begin{array}{c}\mathrm{HR}=1.53(1.32-1.77) \\
I^{2}=0.0 \%\end{array}$ & $\begin{array}{c}\mathrm{HR}=1.76(1.35-2.30) \\
I^{2}=0.5 \%\end{array}$ & $\begin{array}{c}\mathrm{HR}=1.75(0.59-5.17) \\
I^{2}=66.2 \%\end{array}$ & $\begin{array}{c}\mathrm{HR}=1.11(0.79-1.56) \\
I^{2}=0.0 \%\end{array}$ \\
\hline \multicolumn{5}{|l|}{ Cut-off point } \\
\hline$>150$ & $\begin{array}{c}\mathrm{HR}=1.60(1.18-2.17) \\
I^{2}=66.7 \%\end{array}$ & $\begin{array}{c}\mathrm{HR}=2.03(0.73-5.62) \\
I^{2}=86.0 \%\end{array}$ & $\begin{array}{c}\mathrm{HR}=1.22(0.93-1.59) \\
I^{2}=0.0 \%\end{array}$ & $\begin{array}{c}\mathrm{HR}=1.38(1.09-1.74) \\
I^{2}=20.7 \%\end{array}$ \\
\hline$\leq 150$ & $\begin{array}{c}\mathrm{HR}=1.33(1.08-1.64) \\
I^{2}=58.0 \%\end{array}$ & $\begin{array}{c}\mathrm{HR}=1.49(1.03-2.14) \\
I^{2}=71.0 \%\end{array}$ & $\begin{array}{c}\mathrm{HR}=1.34(1.12-1.59) \\
I^{2}=37.8 \%\end{array}$ & / \\
\hline \multicolumn{5}{|l|}{ Country } \\
\hline Asia & $\begin{array}{c}\mathrm{HR}=1.41(1.22-1.63) \\
I^{2}=45.2 \%\end{array}$ & $\begin{array}{c}\mathrm{HR}=1.71(0.97-3.01) \\
I^{2}=73.9 \%\end{array}$ & $\begin{array}{c}\mathrm{HR}=1.75(0.59-5.17) \\
I^{2}=66.2 \%\end{array}$ & I \\
\hline $\begin{array}{l}\text { Europe \& } \\
\text { America }\end{array}$ & $\begin{array}{c}\mathrm{HR}=1.46(1.11-1.91) \\
I^{2}=71.9 \%\end{array}$ & $\begin{array}{c}\mathrm{HR}=1.62(0.99-2.65) \\
I^{2}=79.0 \%\end{array}$ & $\begin{array}{c}\mathrm{HR}=1.30(1.11-1.52) \\
I^{2}=0.0 \%\end{array}$ & $\begin{array}{c}\mathrm{HR}=1.49(1.11-2.00) \\
I^{2}=35.2 \%\end{array}$ \\
\hline \multicolumn{5}{|l|}{ Study quality } \\
\hline$\geq 6$ & $\begin{array}{c}\mathrm{HR}=1.44(1.18-1.77) \\
I^{2}=65.9 \%\end{array}$ & $\begin{array}{c}\mathrm{HR}=1.72(1.17-2.52) \\
I^{2}=76.7 \%\end{array}$ & $\begin{array}{c}\mathrm{HR}=1.30(1.12-1.51) \\
I^{2}=0.0 \%\end{array}$ & $\begin{array}{c}\mathrm{HR}=1.46(1.14-1.87) \\
I^{2}=0.0 \%\end{array}$ \\
\hline$<6$ & $\begin{array}{c}\mathrm{HR}=1.54(1.23-1.93) \\
I^{2}=0.0 \%\end{array}$ & l & l & l \\
\hline \multicolumn{5}{|l|}{$\begin{array}{l}\text { Study analysis } \\
\text { type }\end{array}$} \\
\hline Univariable type & $\begin{array}{c}\mathrm{HR}=1.63(1.29-2.04) \\
I^{2}=57.6 \%\end{array}$ & $\begin{array}{c}\mathrm{HR}=2.08(1.28-3.38) \\
I^{2}=60.6 \%\end{array}$ & $\begin{array}{c}\mathrm{HR}=1.31(1.10-1.56) \\
I^{2}=0.0 \%\end{array}$ & $\begin{array}{c}\mathrm{HR}=1.23(0.60-2.53) \\
I^{2}=63.3 \%\end{array}$ \\
\hline $\begin{array}{l}\text { Multivariable } \\
\text { type }\end{array}$ & $\begin{array}{c}\mathrm{HR}=1.32(1.03-1.69) \\
I^{2}=59.9 \%\end{array}$ & $\begin{array}{c}\mathrm{HR}=1.33(0.91-1.93) \\
I^{2}=66.6 \%\end{array}$ & $\begin{array}{c}\mathrm{HR}=1.28(0.99-1.66) \\
I^{2}=32.7 \%\end{array}$ & $\begin{array}{c}\mathrm{HR}=1.38(1.03-1.85) \\
I^{2}=5.3 \%\end{array}$ \\
\hline
\end{tabular}

HR: hazard ratio; "l" symbol: no results due to insufficient studies.

quality were not significant sources of heterogeneity and did not obviously dominate present results (Table 3 ).

\section{Discussion}

$\mathrm{CRC}$ is a global health problem with a high rate of recurrence and metastasis [1]. Thus, there is an urgent need to explore additional prognostic markers to facilitate earlier and optimized treatment for CRC. Recently, many studies have been performed to assess the clinicopathological and prognostic values of PLR in CRC [8-12]. However, to date, there is still no general agreement on the clinical value of PLR in CRC.

Our results indicated that elevated PLR predicted poor survival in CRC, including OS, DFS, CSS, and RFS. In addition, our results also indicated that elevated PLR was associated with poor tumor stage, $\mathrm{pT}$ category, and degree of differentiation and suggested that PLR may be feasible for tumor staging in CRC. Similar results were obtained in the subgroup analyses.

Several studies have reported that host-derived inflammation, immune response, and coagulation status played an important role in tumor proliferation, invasion, angiogenesis, and metastasis [2-4]. In cancer, the systemic inflammatory response may be secondary to tumor hypoxia or local tissue damage [29] and resulted in an imbalance of immune response, promoting tumor progression [3-5]. As circulating biomarkers for inflammation, immune response, and coagulation status, platelet and lymphocyte counts were reported to be associated with prognosis in CRC $[18,30]$. Thus, we conducted the present study to assess the clinical values of PLR in CRC, and the results indicated that elevated PLR could predict a poor prognosis in CRC. 




(a)

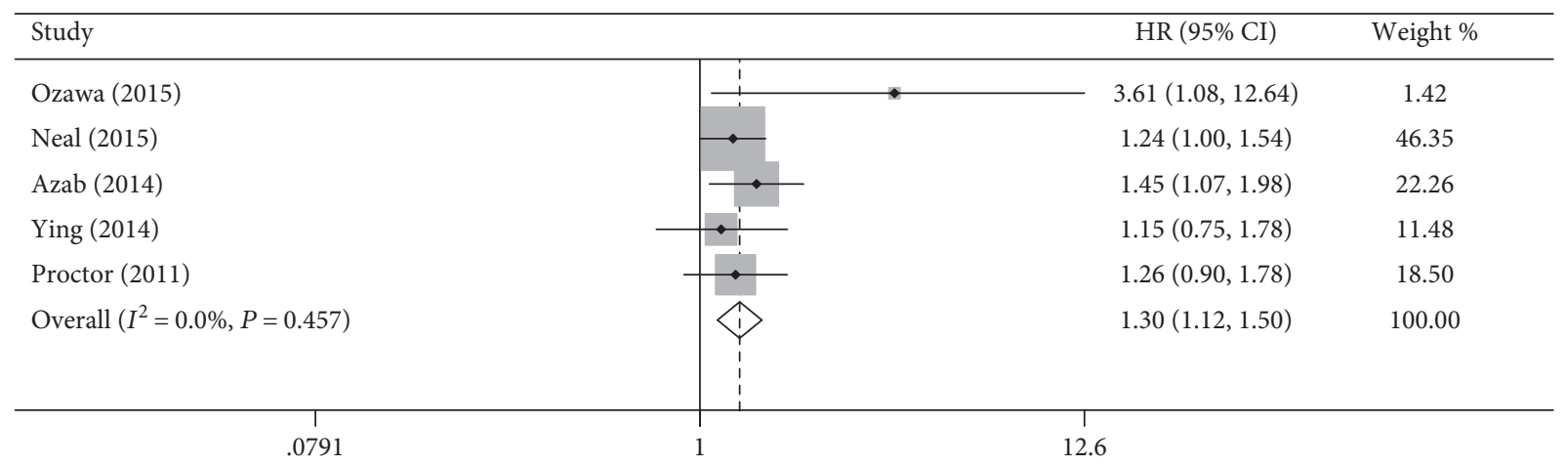

(b)

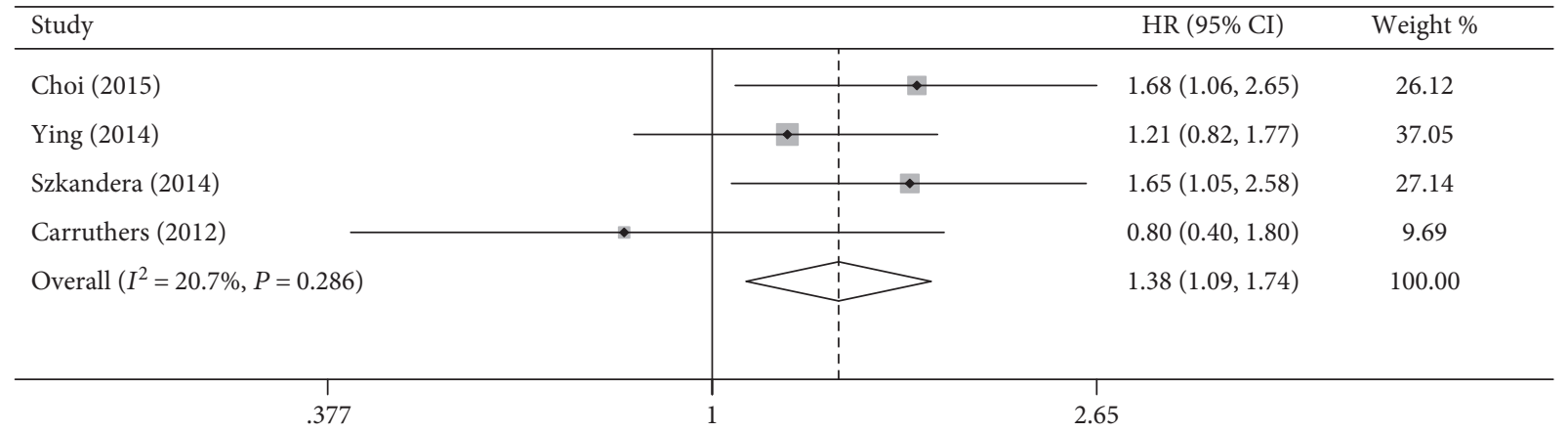

(c)

FIGURE 3: The estimated hazard ratio (HR) was summarized for the relationship between platelet-to-lymphocyte ratio and disease-free survival (a), between platelet-to-lymphocyte ratio and cancer-specific survival (b), and between platelet-to-lymphocyte ratio and recurrence-free survival (c).

The underlying mechanisms responsible for the role of PLR in CRC have not yet been elucidated, but recent experimental and clinical data may provide several potential explanations. An elevated PLR represents an increased number of platelets and/or a decreased number of lymphocytes, and elevated platelets could promote metastatic potential of tumor cells in several biological pathways. Platelets could secrete cellular growth factors (i.e., platelet-derived growth factor, vascular endothelial growth factor, transforming growth factor beta, platelet factor 4 , and inflammatory mediators) and then stimulate tumor angiogenesis and growth $[31,32]$. Besides, several studies have shown that platelets can activate the invasiveness of tumor cells by enhancing the formation of tumor stroma and supporting the stable adhesion of tumor cells to the endothelium [33, 34]. Furthermore, in the bloodstream, the interactions between tumor cells and platelets could facilitate tumor cell metastasis by impeding the clearance of tumor cells by innate immune cells $[34,35]$. Thus, many studies were performed to explore the antitumor activity of antiplatelet agents. Indeed, Suzuki et al. reported that antiplatelet drugs (i.e., cilostazol and prostaglandin I2) could inhibit invasiveness of tumor cells [33], and Mikami et al. showed that antiplatelet antibody or aspirin could inhibit proliferation of tumor cells both in vivo and in vitro [36]. Moreover, several clinical trials demonstrated that aspirin use was associated with 
Begg's funnel plot with pseudo 95\% confidence limits

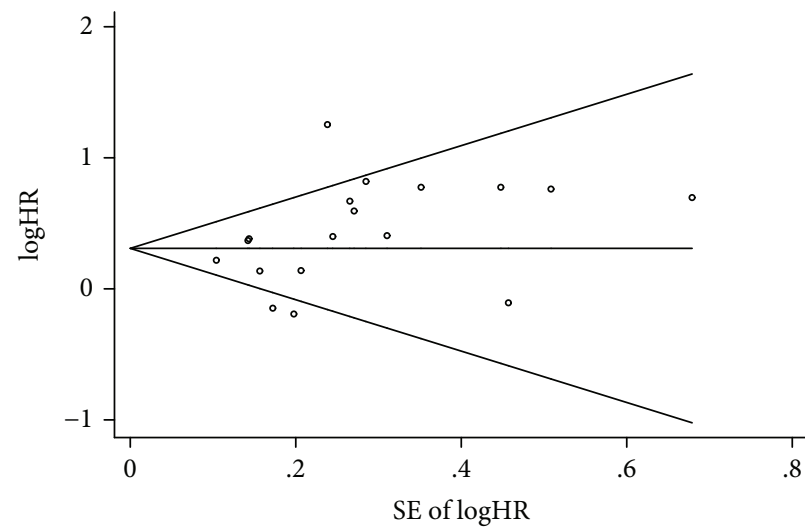

(a)

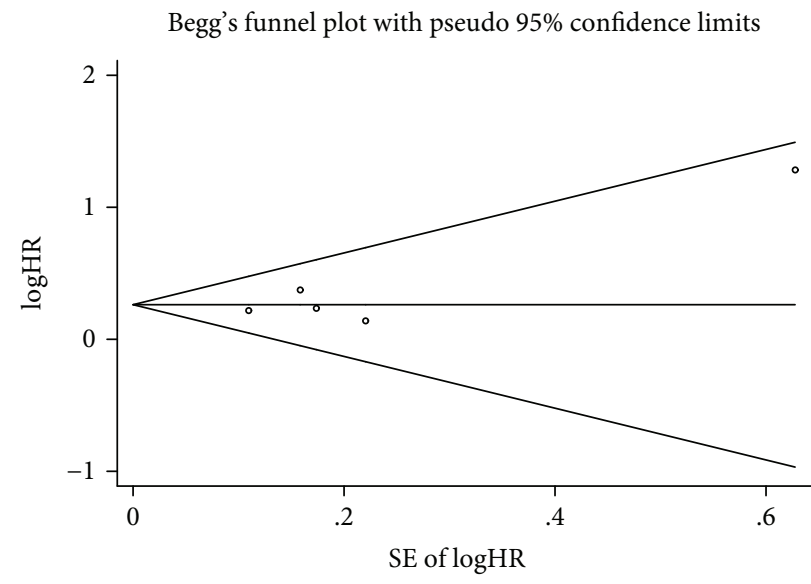

(c)
Begg's funnel plot with pseudo 95\% confidence limits



(b)



(d)

FIGURE 4: Funnel plots assessing publication bias for overall survival (a), disease-free survival (b), cancer-specific survival (c), and recurrencefree survival (d).

lower mortality in CRC $[37,38]$. Future studies are needed to explore the tailored treatments that directly target platelets for the improvement of survival in CRC.

A growing body of evidence reported that lymphocytes could induce apoptosis of tumor cells and were inversely related with tumor proliferation and invasiveness [30,39]. Therefore, a decreased number of lymphocytes could impede antitumor immune response and further facilitate tumor metastatic potential [4]. Several studies also showed that low-tumor-infiltrating lymphocytes are significantly associated with poor survival in CRC $[40,41]$. Accordingly, a systemic review and meta-analysis by Gooden et al. included 10 studies with 3984 CRC patients and indicated that tumorinfiltrating lymphocytes could influence prognosis of CRC [42]. Studies on immunotherapy targeting immune checkpoint (i.e., cytotoxic T-lymphocyte-associated antigen 4 and programmed death 1 receptor) have raised the prospect that the immune system may represent a favorable approach for advancing the treatment of CRC [43, 44]. Further studies are needed to explore the antitumor activity of the host immunity via immunotherapy, especially for subpopulations with lymphopenia.
There were few studies primarily focused on the clinicopathological value of PLR in CRC. In the included studies, we found that the PLR was most frequently elevated in poor clinicopathological characteristics. Accordingly, Azab et al. included patients with stage I to stage IV and the patients were categorized into an equal tertile based on PLR, and results showed that PLR was significantly related with tumor stage $(P<0.001)[17]$. Ying et al. and Choi et al. showed a significant association between PLR and tumor stage and pT category $(P<0.05)[9,12]$. Moreover, Kown et al. reported patients with greater PLR showed an increased likelihood of positive lymph node ratio $>0.2(P=0.0006)$ and a lower 5 -year OS $(P=0.001)$ [20]. Ozawa et al. also found that patients with a high PLR more frequently suffered obstruction or perforation/penetration and on average had larger tumors compared to those with a low PLR [24]. After pooling all relevant studies, our results also suggested that elevated PLR was associated with poor clinicopathological characteristics (i.e., tumor stage, pT category, and degree of differentiation), suggesting that PLR may be feasible for tumor staging. Thus, according to the above results, greater PLR may reflect an enhanced host inflammatory response to more aggressive 
TABLE 3: Metaregression analysis exploring sources of heterogeneity.

\begin{tabular}{lcccc}
\hline & Coefficient & $\begin{array}{c}\text { Standard } \\
\text { error }\end{array}$ & $P$ & $\begin{array}{c}\text { Adjusted } \\
R^{2}\end{array}$ \\
\hline Overall survival & & & & \\
Sampling time & 0.2797 & 0.1995 & 0.18 & $4.16 \%$ \\
Metastatic status & -0.0362 & 0.1089 & 0.744 & $-11.16 \%$ \\
Sample size & -0.0006 & 0.0006 & 0.401 & $2.35 \%$ \\
Cut-off value & 0.0020 & 0.0016 & 0.229 & $23.59 \%$ \\
Country & 0.0277 & 0.1945 & 0.889 & $-11.56 \%$ \\
Study quality & -0.0316 & 0.1196 & 0.795 & $-9.52 \%$ \\
Disease-free & & & & \\
survival & & & & \\
Sampling time & 0.2377 & 0.4092 & 0.579 & $-14.75 \%$ \\
Metastatic status & -0.0380 & 0.2159 & 0.865 & $-19.67 \%$ \\
Sample size & -0.0004 & 0.0011 & 0.739 & $-14.88 \%$ \\
Cut-off value & 0.0017 & 0.0028 & 0.566 & $-7.16 \%$ \\
Country & 0.0376 & 0.3659 & 0.921 & $-19.62 \%$ \\
Study quality & 0.0832 & 0.2664 & 0.764 & $-17.38 \%$ \\
\hline
\end{tabular}

Note: the dependent variable is the lnHR for overall survival or disease-free survival from each study; weights have been assigned according to the estimated variance of the lnHR; cancer-specific survival and recurrencefree survival were not analyzed due to a limited number of studies.

tumor biology and higher tumor burden. Future studies should thoroughly evaluate the association between PLR and clinicopathological characteristics for further providing an additional basis for CRC staging.

The definition for the optimal cut-off value of PLR is urgently required and is the main concern for the clinical utility. There is no agreement on the optimal cut-off value, although most included studies defined a cut-off value of 150. It is unclear whether this cut-off value was appropriate for predicting prognosis in all CRC patients. Song et al., Neal et al., and Kwon et al. grouped PLR into three groups $(<150$, 150-300, and $>300$ ), and the results reported that PLR was significantly associated with OS $[20,22,26]$. Ozawa et al., Neofytou et al., Ying et al., and Szkandera et al. applied a receiver operating curve to calculate the optimal cut-off value, but the optimal cut-off value still varied $(25.4,150$, 175 , and 225 , respectively) $[12,23,24,28]$. The differences of cut-off values may be attributed to the differences of included patients. Indeed, Ozawa et al., Neofytou et al., Ying et al., and Szkandera et al. included stage II CRC, colorectal liver metastasis, stages I-III CRC and II-III colon cancer, respectively. Moreover, Kwon et al. and Song et al. both reported that $<15 \%$ patients were grouped into the subset of the highest tertile PLR > $300[20,26]$. Therefore, it was notable that a high cut-off value may lead to the omission of a greater number of patients in clinical practice although it may be more valuable. Further studies are needed to explore whether the optimal cut-off values of PLR differ among different population and then to define the optimal cut-off value of PLR for future individual treatments.

Although previous meta-analyses evaluated the prognostic values of PLR for CRC, the cut-off value of PLR and the association between PLR and clinicopathological characteristics were not assessed $[45,46]$. Thus, this study had several obvious advantages. First, our study included more eligible studies, making our results more powerful and robust. Second, our studies assessed the impact of cut-off values on prognostic values. Third, we found that the PLR was most frequently elevated in advanced stage tumor for CRC, and we assessed the prognostic role of PLR in metastasis positive and negative groups using rational and robust subgroups. In addition, we assessed the quality of included studies and then performed subgroup analyses based on the study quality.

There were several limitations in the present study. First, our study was based on the published data. Several HRs with 95\% CIs were calculated from available data in the studies that did not provide HRs directly. Second, considerable heterogeneity existed in the present study, and we used a relatively conservative random effects model if there was heterogeneity significant; and, therefore, it may underestimate the prognostic value of PLR in CRC. Although our metaregression analysis did not found significant sources of heterogeneity, the heterogeneity could be also caused by differences in patient characteristics (i.e., age, gender, race, and treatment strategies) and thus heterogeneity could not be completely eliminated. Therefore, further large-scale multicenter studies on homogeneous patients and diagnostic method are required to investigate the prognostic values of PLR in CRC. Moreover, our study did not provide results regarding the optimal cut-off value and whether the cut-off values differed in the assessment of clinicopathological characteristics and prognosis values. In addition, although we performed subgroup analysis based on analysis type in the primary studies (multivariable and univariable analysis), the variables that were included in multivariable analysis or adjustment were different in the primary studies and the number of included studies was also limited, and thus, we could not conducted in-depth subgroup analysis based on the various variables. Further studies are needed to assess the prognostic values of PLR in CRC using optimal multivariable analysis or adjustment.

\section{Conclusions}

Our results indicate that elevated PLR predicted poor prognosis and clinicopathological characteristics in CRC. PLR is a convenient and low-cost approach for the prognostic prediction and individualized treatment for CRC. Future studies are required to identify the optimal cut-off value of PLR and improve the clinical utility of PLR.

\section{Additional Points}

The corresponding author had full access to all the data and analyses.

\section{Conflicts of Interest}

The authors declare that there is no conflict of interest regarding the publication of this paper. 


\section{Acknowledgments}

The authors thank the Department of Chemotherapy and Radiotherapy of Second Affiliated Hospital and Yuying Children's Hospital of Wenzhou Medical University for the technical assistance.

\section{References}

[1] L. A. Torre, F. Bray, R. L. Siegel, J. Ferlay, J. Lortet-Tieulent, and A. Jemal, "Global cancer statistics, 2012," CA: A Cancer Journal for Clinicians, vol. 65, no. 2, pp. 87-108, 2015.

[2] L. M. Coussens and Z. Werb, "Inflammation and cancer," Nature, vol. 420, no. 6917, pp. 860-867, 2002.

[3] D. G. DeNardo and L. M. Coussens, "Inflammation and breast cancer. Balancing immune response: Crosstalk between adaptive and innate immune cells during breast cancer progression," Breast Cancer Research, vol. 9, no. 4, p. 212, 2007.

[4] G. P. Dunn, L. J. Old, and R. D. Schreiber, "The immunobiology of cancer immunosurveillance and immunoediting," Immunity, vol. 21, no. 2, pp. 137-148, 2004.

[5] E. Elinav, R. Nowarski, C. A. Thaiss, B. Hu, C. Jin, and R. A. Flavell, "Inflammation-induced cancer: crosstalk between tumours, immune cells and microorganisms," Nature Reviews Cancer, vol. 13, no. 11, pp. 759-771, 2013.

[6] G. Qiang, C. Liang, F. Xiao et al., "Prognostic significance of platelet-to-lymphocyte ratio in non-small-cell lung cancer: a meta-analysis," OncoTargets and Therapy, vol. 9, pp. 869876, 2016.

[7] S. Krenn-Pilko, U. Langsenlehner, E. M. Thurner et al., “The elevated preoperative platelet-to-lymphocyte ratio predicts poor prognosis in breast cancer patients," British Journal of Cancer, vol. 110, no. 10, pp. 2524-2530, 2014.

[8] W. He, C. Yin, G. Guo et al., "Initial neutrophil lymphocyte ratio is superior to platelet lymphocyte ratio as an adverse prognostic and predictive factor in metastatic colorectal cancer," Medical Oncology (Northwood, London, England), vol. 30, no. 1, p. 439, 2013.

[9] W. J. Choi, M. C. Cleghorn, H. Jiang, T. D. Jackson, A. Okrainec, and F. A. Quereshy, "Preoperative neutrophil-to-lymphocyte ratio is a better prognostic serum biomarker than plateletto-lymphocyte ratio in patients undergoing resection for nonmetastatic colorectal cancer," Annals of Surgical Oncology, vol. 22 Supplement 3, pp. 603-613, 2015.

[10] Z. M. Li, Y. F. Peng, C. Z. Du, and J. Gu, "Colon cancer with unresectable synchronous metastases: the AAAP scoring system for predicting the outcome after primary tumour resection," Colorectal Disease, vol. 18, no. 3, pp. 255-263, 2016.

[11] H. J. Son, J. W. Park, H. J. Chang et al., "Preoperative plasma hyperfibrinogenemia is predictive of poor prognosis in patients with nonmetastatic colon cancer," Annals of Surgical Oncology, vol. 20, no. 9, pp. 2908-2913, 2013.

[12] H. Q. Ying, Q. W. Deng, B. S. He et al., "The prognostic value of preoperative NLR, d-NLR, PLR and LMR for predicting clinical outcome in surgical colorectal cancer patients," Medical Oncology (Northwood, London, England), vol. 31, no. 12, p. 305, 2014.

[13] A. Stang, "Critical evaluation of the Newcastle-Ottawa scale for the assessment of the quality of nonrandomized studies in meta-analyses," European Journal of Epidemiology, vol. 25, no. 9, pp. 603-605, 2010.
[14] J. F. Tierney, L. A. Stewart, D. Ghersi, S. Burdett, and M. R. Sydes, "Practical methods for incorporating summary time-to-event data into meta-analysis," Trials, vol. 8, no. 1, p. 16, 2007.

[15] C. H. Schmid, P. C. Stark, J. A. Berlin, P. Landais, and J. Lau, "Meta-regression detected associations between heterogeneous treatment effects and study-level, but not patient-level, factors," Journal of Clinical Epidemiology, vol. 57, no. 7, pp. 683-697, 2004.

[16] S. Duval and R. Tweedie, "Trim and fill: a simple funnelplot-based method of testing and adjusting for publication bias in meta-analysis," Biometrics, vol. 56, no. 2, pp. 455463, 2000.

[17] B. Azab, F. Mohammad, N. Shah et al., "The value of the pretreatment neutrophil lymphocyte ratio vs. platelet lymphocyte ratio in predicting the long-term survival in colorectal cancer," Cancer Biomarkers, vol. 14, no. 5, pp. 303-312, 2014.

[18] Z. Baranyai, M. Krzystanek, V. Josa et al., "The comparison of thrombocytosis and platelet-lymphocyte ratio as potential prognostic markers in colorectal cancer," Thrombosis and Haemostasis, vol. 111, no. 3, pp. 483-490, 2014.

[19] R. Carruthers, L. M. Tho, J. Brown, S. Kakumanu, E. McCartney, and A. C. McDonald, "Systemic inflammatory response is a predictor of outcome in patients undergoing preoperative chemoradiation for locally advanced rectal cancer," Colorectal Disease, vol. 14, no. 10, pp. e701-e707, 2012.

[20] H. C. Kwon, S. H. Kim, S. Y. Oh et al., "Clinical significance of preoperative neutrophil-lymphocyte versus plateletlymphocyte ratio in patients with operable colorectal cancer," Biomarkers, vol. 17, no. 3, pp. 216-222, 2012.

[21] K. Mori, Y. Toiyama, S. Saigusa et al., "Systemic analysis of predictive biomarkers for recurrence in colorectal cancer patients treated with curative surgery," Digestive Diseases and Sciences, vol. 60, no. 8, pp. 2477-2487, 2015.

[22] C. P. Neal, V. Cairns, M. J. Jones et al., "Prognostic performance of inflammation-based prognostic indices in patients with resectable colorectal liver metastases," Medical Oncology (Northwood, London, England), vol. 32, no. 5, p. 144, 2015.

[23] K. Neofytou, E. C. Smyth, A. Giakoustidis, A. Z. Khan, D. Cunningham, and S. Mudan, "Elevated platelet to lymphocyte ratio predicts poor prognosis after hepatectomy for liver-only colorectal metastases, and it is superior to neutrophil to lymphocyte ratio as an adverse prognostic factor," Medical Oncology (Northwood, London, England), vol. 31, no. 10, p. 239, 2014.

[24] T. Ozawa, S. Ishihara, T. Nishikawa et al., "The preoperative platelet to lymphocyte ratio is a prognostic marker in patients with stage II colorectal cancer," International Journal of Colorectal Disease, vol. 30, no. 9, pp. 1165-1171, 2015.

[25] M. J. Proctor, D. S. Morrison, D. Talwar et al., “A comparison of inflammation-based prognostic scores in patients with cancer. A Glasgow Inflammation Outcome Study," European Journal of Cancer (Oxford, England : 1990), vol. 47, no. 17, pp. 2633-2641, 2011.

[26] A. Song, W. Eo, and S. Lee, "Comparison of selected inflammation-based prognostic markers in relapsed or refractory metastatic colorectal cancer patients," World Journal of Gastroenterology, vol. 21, no. 43, pp. 12410-12420, 2015.

[27] Z. Q. Sun, X. N. Han, H. J. Wang et al., "Prognostic significance of preoperative fibrinogen in patients with colon cancer," World Journal of Gastroenterology, vol. 20, no. 26, pp. 8583-8591, 2014. 
[28] J. Szkandera, M. Pichler, G. Absenger et al., "The elevated preoperative platelet to lymphocyte ratio predicts decreased time to recurrence in colon cancer patients," American Journal of Surgery, vol. 208, no. 2, pp. 210-214, 2014.

[29] C. S. Roxburgh and D. C. McMillan, "Role of systemic inflammatory response in predicting survival in patients with primary operable cancer," Future Oncology (London, England), vol. 6, no. 1, pp. 149-163, 2010.

[30] L. Liang, J. Zhu, H. Jia et al., "Predictive value of pretreatment lymphocyte count in stage II colorectal cancer and in high-risk patients treated with adjuvant chemotherapy," Oncotarget, vol. 7, no. 1, pp. 1014-1028, 2016.

[31] S. A. Kono, L. E. Heasley, R. C. Doebele, and D. R. Camidge, "Adding to the mix: fibroblast growth factor and plateletderived growth factor receptor pathways as targets in nonsmall cell lung cancer," Current Cancer Drug Targets, vol. 12, no. 2, pp. 107-123, 2012.

[32] H. M. Verheul, K. Hoekman, F. Lupu et al., "Platelet and coagulation activation with vascular endothelial growth factor generation in soft tissue sarcomas," Clinical Cancer Research, vol. 6, no. 1, pp. 166-171, 2000.

[33] K. Suzuki, K. Aiura, M. Ueda, and M. Kitajima, “The influence of platelets on the promotion of invasion by tumor cells and inhibition by antiplatelet agents," Pancreas, vol. 29, no. 2, pp. 132-140, 2004.

[34] J. S. Palumbo, K. E. Talmage, J. V. Massari et al., "Platelets and fibrin(ogen) increase metastatic potential by impeding natural killer cell-mediated elimination of tumor cells," Blood, vol. 105, no. 1, pp. 178-185, 2005.

[35] B. Nieswandt, M. Hafner, B. Echtenacher, and D. N. Männel, "Lysis of tumor cells by natural killer cells in mice is impeded by platelets," Cancer Research, vol. 59, no. 6, pp. 1295-1300, 1999.

[36] J. Mikami, Y. Kurokawa, T. Takahashi et al., "Antitumor effect of antiplatelet agents in gastric cancer cells: an in vivo and in vitro study," Gastric Cancer, vol. 19, no. 3, pp. 817-826, 2016.

[37] C. McCowan, A. J. Munro, P. T. Donnan, and R. J. Steele, "Use of aspirin post-diagnosis in a cohort of patients with colorectal cancer and its association with all-cause and colorectal cancer specific mortality," European Journal of Cancer (Oxford, England: 1990), vol. 49, no. 5, pp. 1049-1057, 2013.

[38] A. T. Chan, S. Ogino, and C. S. Fuchs, "Aspirin use and survival after diagnosis of colorectal cancer," Jama, vol. 302, no. 6, pp. 649-658, 2009.

[39] J. E. Niederhuber, "Cancer vaccines: the molecular basis for T cell killing of tumor cells," The Oncologist, vol. 2, no. 5, pp. 280-283, 1997.

[40] W. S. Lee, S. Park, W. Y. Lee, S. H. Yun, and H. K. Chun, "Clinical impact of tumor-infiltrating lymphocytes for survival in stage II colon cancer," Cancer, vol. 116, no. 22, pp. 51885199, 2010.

[41] D. M. Frey, R. A. Droeser, C. T. Viehl et al., "High frequency of tumor-infiltrating FOXP3(+) regulatory $\mathrm{T}$ cells predicts improved survival in mismatch repair-proficient colorectal cancer patients," International Journal of Cancer, vol. 126, no. 11, pp. 2635-2643, 2010.

[42] M. J. Gooden, G. H. de Bock, N. Leffers, T. Daemen, and H. W. Nijman, "The prognostic influence of tumour-infiltrating lymphocytes in cancer: a systematic review with meta-analysis," British Journal of Cancer, vol. 105, no. 1, pp. 93-103, 2011.
[43] D. T. Le, J. N. Uram, H. Wang et al., "PD-1 blockade in tumors with mismatch-repair deficiency," The New England Journal of Medicine, vol. 372, no. 26, pp. 2509-2520, 2015.

[44] C. H. Son, J. H. Bae, D. Y. Shin et al., "CTLA-4 blockade enhances antitumor immunity of intratumoral injection of immature dendritic cells into irradiated tumor in a mouse colon cancer model," Journal of Immunotherapy, vol. 37, no. 1, pp. 1-7, 2014.

[45] X. Gu, X. S. Gao, S. Qin et al., "Elevated platelet to lymphocyte ratio is associated with poor survival outcomes in patients with colorectal cancer," PloS One, vol. 11, no. 9, Article ID e0163523, 2016.

[46] H. X. Peng, K. Lin, B. S. He et al., "Platelet-to-lymphocyte ratio could be a promising prognostic biomarker for survival of colorectal cancer: a systematic review and meta-analysis," FEBS Open Bio, vol. 6, no. 7, pp. 742-750, 2016. 




The Scientific World Journal
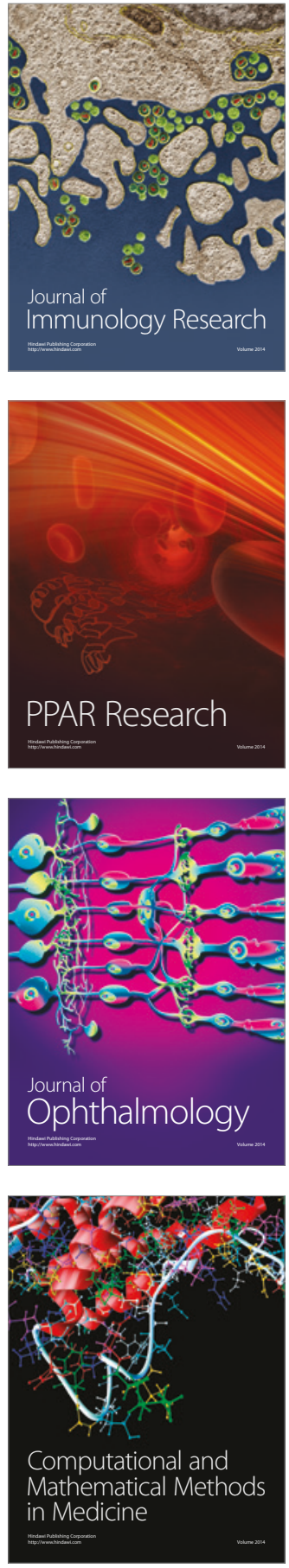

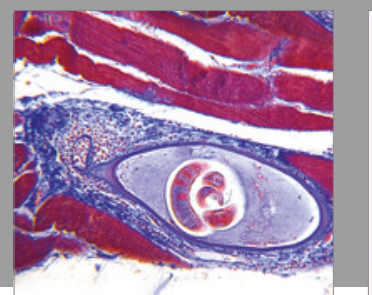

Gastroenterology Research and Practice
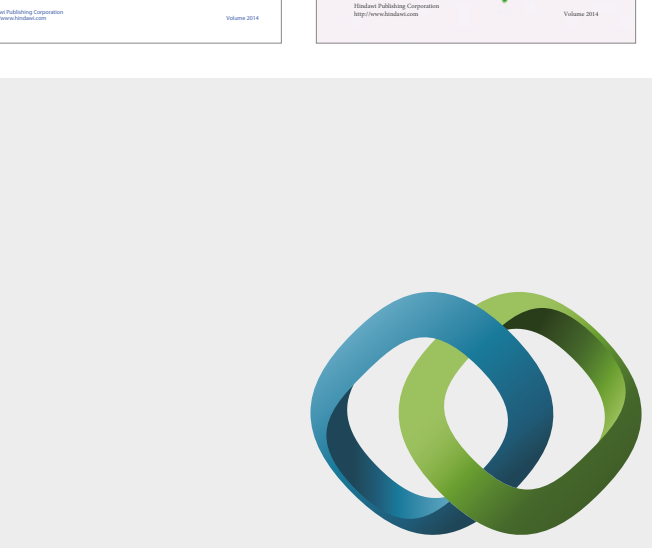

\section{Hindawi}

Submit your manuscripts at

https://www.hindawi.com
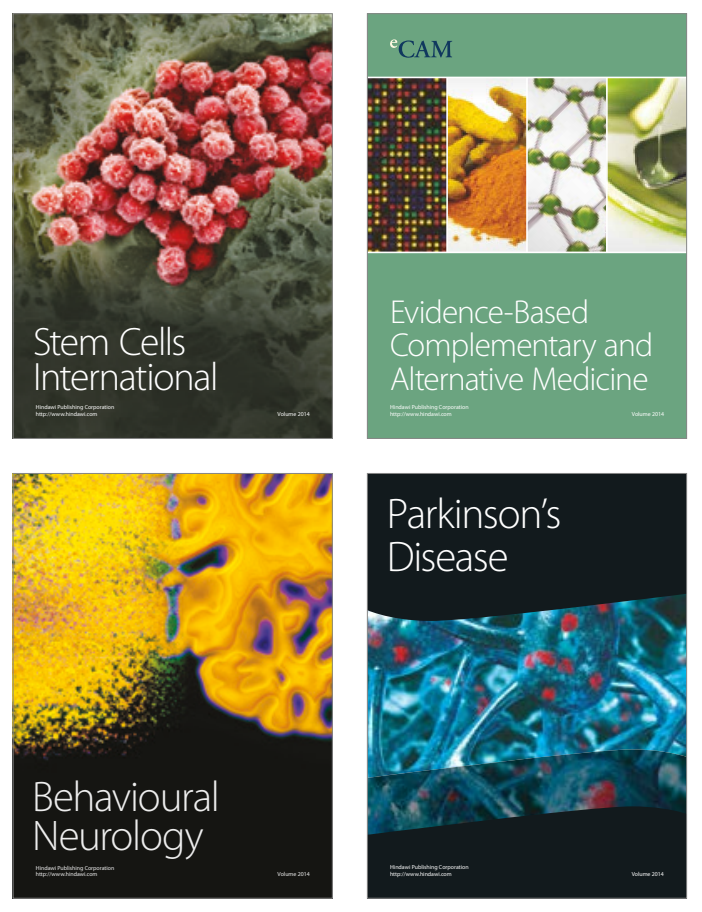
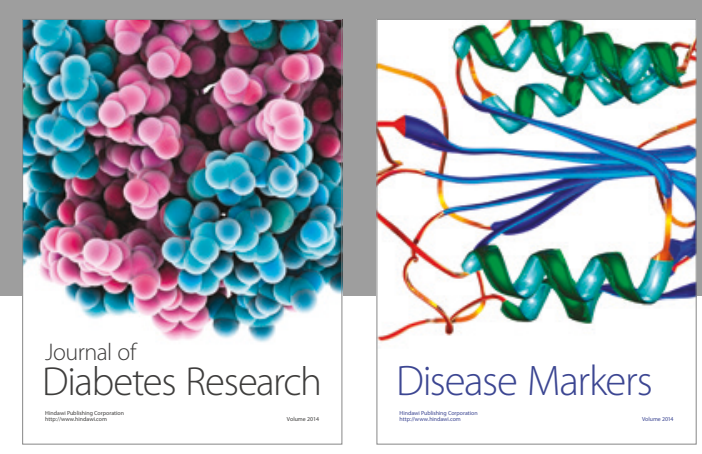

Disease Markers
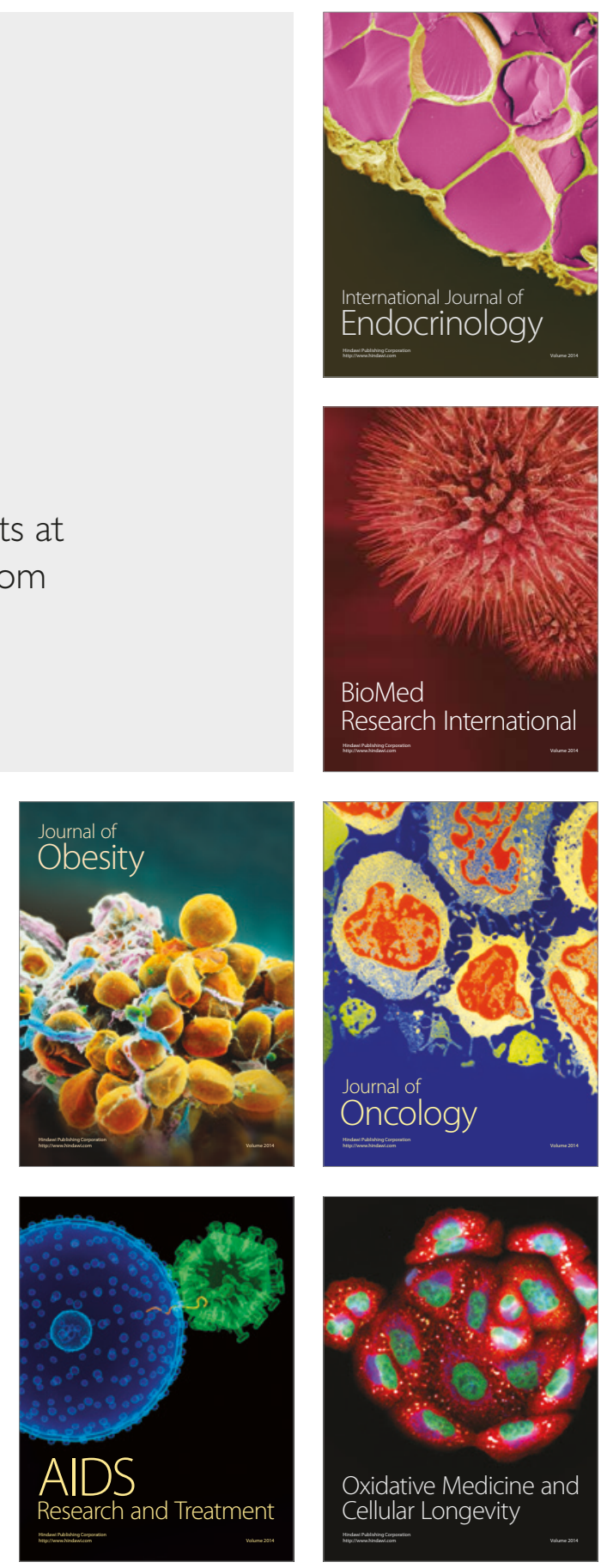